\title{
PETIT PLAIDOYER POUR L'AUTONOMISATION AU NIVEAU SUPÉRIEUR EN FRANÇAIS LANGUE ÉTRANGÈRE
}

\begin{abstract}
Wilczyńska Weronika, Petit plaidoyer pour l'autonomisation au niveau supérieur en français langue étrangère [A short defence speech for autonomization at the advanced level of learning French as a foreign language], Studia Romanica Posnaniensia, Adam Mickiewicz University Press, Poznań, vol. XXVII: 2001, pp. 187-194. ISBN 83-232-1039-X, ISSN 0137-2475.

This contribution starts with arguing, at both theoretical and practical levels, for autonomization in the advanced teaching/learning of French, showing that approach as highly recommendable in the presentday situation in Poland, especially in the university setting. The second part of the article gives evidence of it, referring to a research project conducted recently in this precise context, in which autonomization appeared as very useful also in teacher training.
\end{abstract}

\section{FAUT-IL ENCORE PARLER D'AUTONOMIE?}

Autonomie, autonomisation deviennent des termes tellement à la mode qu'on en voit déjà apparaître des emplois plus ou moins abusifs, avec par exemple une intention purement rhétorique (exploiter la valeur emblématique du terme pour se donner une apparence professionnellement branchée) ou encore une intention humoristique (se donner un prétexte «prestigieux» pour se dispenser de ses obligations d'enseignement)... En fait, de tels effets de mode sont bien caractéristiques de nos pratiques discursives par rapport à des termes à la fois évocateurs et prestigieux, et ce phénomène ne saurait suffire, à lui seul, pour nous pousser à nous occuper - une fois de plus - du fond de la question.

Or, bien que l'autonomisation constitue l'objet de multiples applications de terrain, ainsi que de recherches théoriques et empiriques très poussées, elle n'est pas à l'abri de malentendus bien plus graves, concernant la nature même et la finalité de nos interventions pédagogiques. Ce genre de dérapages est d'autant plus facile que:

- dans les opinions courantes, la DL est considérée - tout comme quelques autres disciplines (médecine, économie, etc.) faisant l'objet d'imaginaires collectifs plus ou moins simplifiés - tantôt comme un ART (reposant sur un talent inné, voire 
sur une vocation), tantôt comme un domaine d'expérience courante (et donc soumis aux seules règles de bon sens) qui en tant que tel reste à la portée de tout le monde aussi bien pour ce qui est de la pratique que sur le plan des jugements et opinions critiques;

- sur le terrain universitaire, la DL a toujours du mal à se constituer et à se faire reconnaître comme non seulement un domaine d'activité pratique et de réflexion de type méthodologique, mais aussi une discipline universitaire à part entière. En résultat, elle continue encore quelquefois d'être perçue comme une spécialité mineure, une soeur cadette de la linguistique - comme en témoigne son statut universitaire actuel la dissimulant sous l'appellation de linguistique appliquée (ce dernier terme fonctionnant, dans les thèses de doctorats polonaises, par exemple, comme dénomination officielle de la spécialité).

Indépendamment de cela, l'autonomisation - comme toute conception nouvelle et populaire à la fois - peut susciter des interrogations portant autant sur sa nature que sur sa valeur pour la didactique et la pédagogie de classe. Parmi les questions les plus fréquentes se trouvent les suivantes: S'agit-il d'une simple tentative de renouveler la DL? S'agit-il d'une nouvelle «méthode» d'enseignement? L'enseignant aura-t-il encore un rôle à jouer dans un apprentissage autonome? Nous pourrions ajouter à cette liste une question plus spécifique, qui concerne directement le contexte de perfectionnement linguistique: Que peut-elle apporter au niveau supérieur qui sous-entend tout autant une spécialisation de discours qu'une diversification importante de situations de communications?

Pour répondre à ce genre de questions, qu'elles soient générales ou spécifiques, il nous semble indispensable de partir de la conception que l'on se fait de l'objet même de la DL. Or, ceci nous oblige à définir au préalable la perspective d'approche - laquelle détermine (comme le remarquait déjà - pour la linguistique - de Saussure) l'objet, la terminologie connexe et la méthodologie de recherche.

Ces précisions seront confrontées avec les résultats d'un projet de recherche mené dans les années 1995-1997 et portant sur l'autonomisation au niveau avancé et mis en oeuvre (faute de financement officiel) de manière autonome! Cette réflexion sera ainsi pour nous une occasion pour porter un regard rétrospectif, à 6 ans de distance, sur le cadre théorique général fondant l'expérience en question.

\section{DIDACTIQUE / PÉDAGOGIE: OBJET ET RELATIONS RÉCIPROQUES}

Couramment, on admet que les deux domaines s'intéressent à un même objet, avec une approche respectivement théorique et pratique. Mais ...quel est donc cet objet?

En fait, il semble que les réserves que suscite souvent dans les milieux d'enseignants et d'apprenants l'idée même d'autonomie soient générées par la conception traditionnelle de l'objet de la didactique/pédagogie, conception qui 
impose la manière d'envisager ces deux domaines selon une logique de transmission de connaissances:

\section{Egt $\rightarrow$ objet de connaissance $\rightarrow$ Apt}

La DL repose ici sur une logique «applicationnelle», voire «applicationniste», qui ne va pas sans produire de déceptions du côté des pédagogues comme de celui des linguistes (citons ici un seul exemple d'application «trop fídèle»: l'échec flagrant qu'ont connu les arborescences en grammaire du français langue manternelle). Le chemin parcouru depuis nous pousse à agir avec bien plus de circonspection: on ne saurait plus admettre que les règles intérieures que se forme l'apprenant soient identiques à celles du linguiste. De même, on ne saurait plus affirmer que la compétence de l'apprenant se construise sur un mode additif, avec pour unité de base des informations (connaissances) et capacités relatives à différentes règles...

Or, si le linguiste n'est pas en mesure de pourvoir aux besoins de l'élève, le pédagogue n'est pas forcément mieux loti pour accomplir auprès de l'élève la mission de transfert de données. Car, à la différence de la plus belle fille du monde, l'enseignant ne peut pas tout simplement «donner» ce qu'il a, du moins pour ce qui est des objets complexes - et la compétence de communication en est un par excellence! Qui plus est, un tel don ne serait d'ailleurs pas souhaitable car pour l'étudiant il ne s'agit aucunement de devenir une copie fidèle du maître. Déjà $\mathrm{St}$ Augustion (De magistro) s'interrogeait à ce propos: Quel père serait assez fou pour envoyer son fils à l'école à la seule fin d'apprendre ce que le maître pense? A plus forte raison, au niveau avancé, l'étudiant se doit de développer sa propre manière d'apprendre et de construire sa compétence à communiquer à la mesure de sa personnalité et de ses ambitions.

Ainsi, en adoptant résolument la perspective personnelle (celle de l'apprenant), la didactique autonomisante ne fait pas simplement un choix «méthodologique» elle prend acte de la nature individuelle de l'apprentissage en y reconnaissant un processus personnel dans lequel nul ne saurait remplacer le principal interessé, c.-à-d. l'apprenant lui-même. Or, une telle option fait entrevoir tout autrement la finalité de la relation pédagogique et de sa nature:

\section{l'objet de la DL = l'enseignement / apprentissage $\rightarrow$ une compétence à communiquer en LE}

où l'orientation vers une compétence personnelle est essentielle. Or, à la différence d'un corps de connaissances et/ou de capacités fixe et déterminé par avance, une compétence, correspond à un ensemble intégré d'apprentissages personnalisés et dynamiques, suceptible de se modifer au fil de finalités, de situations et de réalisations déterminées du point de vue du sujet.

La relation enseignement $\rightarrow$ apprentissage est à interpréter comme l'accroissement de chances pour qu'un apprentissage se produise, dans le sens de le rendre possible, de le faciliter, de le susciter, etc. L'enseignement et l'apprentissage 
n'y sont plus considẻrés comme les deux «côtés» de la relation pédagogique, supposés plus ou moins symétriques et entretenant en plus entre eux une relation de cause à effet.

Il est clair que les deux visions de l'objet débouchent sur des didactiques / pédagogies très différentes. La première comporte un risque très réel d'une didactisation excessive de l'objet à apprendre, la seconde vise l'économie et l'efficacité de l'apprentissage, telles qu'elles se définissent du point de vue de l'apprenant.

\section{AUTONOMISATION: NÉCESSITÉ AU NIVEAU DIDACTIQUE}

Il existe beaucoup de raisons pour développer l'autonomie de chacun, celle de l'étudiant, comme celle de l'enseignant, d'ailleurs... Nous nous limiterons à évoquer ici un seul argument en faveur de la dẻmarche autonomisante: apprendre à communiquer dans une LE est une tâche trop complexe pour se baser sur le trio classique: analyse - mémorisation - réemploi, trio qui fonde la pédagogie dite traditionnelle et qui néanmoins règne dans bien de nos classes et salles d'université, ceci sans tenir compte de l'expérience courante qui montre à l'évidence que, face à la communication rélle, les étudiants n'arrivent pas très souvent, pour reprendre ici leur propre formulation, à gérer simultanément la forme et le fond!

Pour le rendre à même de maitriser la communication sous tous ces aspects, il n'est point de chemin court et efficace à la fois. La seule formule réaliste et économique consiste, selon nous, à le faire s'initier progressivement à la gestion online de données multiples, en l'amenant à harmoniser - à sa manière et pour son compte - trois types de données, qui sont à la fois, selon nous, les trois piliers de succès:

\section{A. Un esprit commun à partager}

L'enseignant et l'apprenant devraient s'accorder sur la manière d'envisager les notions de base qui forment le cadre de leur action conjointe. Parmi celles-ci il convient de citer les idées suivantes:

- l'activité de l'étudiant est essentielle: tout se fait POUR lui, mais aussi PAR lui; par conséquent l'étudiant ne devrait pas se considérer comme un rouage «fonctionnant» dans la machine de formation, mais comme un sujet à part entière dans le sens de vivre et d'asssumer la (co)responsabilité de son apprentissage et de ses résultats;

- l'objet - compétence(s) en communication en $L E$ - exige une pratique personnelle (donc, en ce sens, authentique) et éclairée, d'où la nécesssité de construire cette compétence en fonction de ce qu'on est et ce qu'on se propose de devenir (le plan de l'identité personnelle et celui de l'auto-accomplissement;

- le rôle de l'enseignant s'assimile à celui d'un guide: quelqu'un qui voit plus loin que l'apprenant mais ne saurait se subsituter à lui; une telle distribution de 
rôles doit conduire l'étudiant à rompre avec le syndrome de la passivité: Me voici! Enseignez-moi! ${ }^{1}$, attitude de repli sur soi-même et de non-action personnelle: pas d'objectifs personnels, pas d'initiative au niveau du comment, pas plus que de responsabilité au nivau des résultats...

Même si cette liste n'est aucunement exclusive, elle constitue une ébauche de ce que peut devenir la plate-forme de coopération pédagogique de l'enseignant et de l'apprenant.

\section{B. Apprendre à apprendre}

Demander tout simplement de maîtriser différentes régularités de la LE, sans aider l'apprenant pour ce qui est du comment le faire, nous semble douteux du point de vue éthique tout autant qu'inefficace avec ceux qui n'ont pas été capables de développer - pour quelque raison que ce soit - d'habitudes et de stratégies personnelles adéquates.

C. Savoir s'organiser pour mieux apprendre passera par:

- une prise de conscience quant à son style cognitif, ses habitudes / préférences de perception, de mémorisation, de restitution, etc.

- une exploitation personnellement optimale de ses caractéristiques dans des activités de découverte et d'appropriation de régularités du système étranger;

- organisation pratique du travail d'apprentissage (en équipe et individuellement, accès à la documentation, différents types d'exposition à la LE);

- une redéfinition des attentes envers l'enseignant: négociations de finalités, de rythmes et de modes d'apprentissage;

- la coopération avec l'enseignant selon le principe d' harmonie pédagogique (cf. Wilczyńska 1999).

\section{Pratique personnelle (authentique)}

L'idée de base est de permettre à l'étudiant d'acquérir de l'expérience à respecter les caractéristiques des différents types de discours à travers des situations de communication personnelles, ce qui suppose une gestion communicationnelle, émotionnelle, intellectuelle, etc. des échanges de différents types. Il conviendra donc de réserver une place de choix à des activités suffisamment représentatives sur un plan typologique (thèmes, situations, partenaires, finalités pragmatiques), tout en les rapportant à la personne de l'apprenant qui sera invité à les gérer dans différentes configurations.

En suivant cette conception, la pédagogie devra s'orienter vers l'exécution de tâches de communication, avec exercices comme activités préparatoires.

\footnotetext{
${ }^{1}$ Cette formule lapidaire proposée par Obrębska (1988), à la suite d'un examen de différentes attitudes de passivité, nous semble bien résumer le point de vue de l'étudiant réduit pendant de longues années au rôle d'enseigné...
} 


\section{EXPÉRIENCE D'AUTONOMISATION: NÉCESSITÉ AU NIVEAU DE LA «RÉALITÉ»}

Sur le plan pratique, la pédagogie d'autonomisation s'est consitituée comme une action d'adaptation à la réalité que notre équipe a dû confronter à partir du «grand tournant» de 1990. Parmi les éléments qui déterminaient la nouvelle situation il convient de retenir notamment:

- le niveau des étudiants en première année, qui devenait de plus en plus hétérogène, ce qui nous a mis dans l'impossibilité de les faire travailler tous de la même manière, selon les mêmes rythmes et en les orientant vers les mêmes objectifs académiques;

- des problèmes d'organisation pratique: les groupes devenus relativement nombreux (de 20 personnes env.), trop peu d'enseignants, préparation d'examens et de tests devenue trop difficile pour les enseignants;

- la nécessité de moderniser nos programmes d'enseignement, et surtout l'urgence de redéfinir les exigences et la pédagogie du FLE appliquées durant les 3 premières années du cursus;

- et enfin, la conscience que, avec la variété accrue de débouchés, la spécialisation individuelle deviendra chose courante et que l'objectif ultime de la formation offerte ne pouvait être autre que de ...se passer de l'enseignant en devenant autonome!

- Il va de soi, qu'avec le changement de la situation, notre équipe a vite mesuré le besoin d'une réorientation méthodologique qui permettrait de faire face à des enjeux nouveaux, tout en dépassant l'ancien modèle de la relation pédagogique fondée sur la transmission de données et la domination de l'enseignant. En effet, l'expérience d'autonomisation a permis:

- d'offrir à un groupe d'enseignants intéressés par cette démarche l'occasion de consolider leurs intuitions quant au rôle fondamental de l'activité authentique de l'apprenant, et d'élaborer des moyens pratiques d'action;

- d'initier les étudiants en maîtrise (didactique FLE) à une expérience de tutorat, combinée à une initiation à la recherche-action, démarche méthodologique qu'ils devaient appliquer par la suite en menant leur propre étude pédagogique dans le cadre de leur travail de maitrise.

C'est précisément cet aspect de la démarche d'autonomisation qui sera décrit brièvement ci-après, à titre d'illustration.

\section{L'EXPÉRIENCE DE TUTORAT}

L'expérience en question a été proposée aux étudiants en première année de maîtrise (didactique FLE). Après avoir entendu et prếparé, au cours du $7^{\mathrm{e}}$ semestre, une série d'exposés ayant pour thème dominant la centration sur l'apprenant, ils 
ont eu l'occasion au cours du semestre suivant, en plus des séances «normales», de s'essayer à influencer les représentations et les pratiques d'apprentissage de leurs tutorés de première année d'études (donc leurs cadets de 3 ans) dans le cadre d'un tutorat bénévole (contacts limités à des rencontres hebdomadaires d'une demiheure!). Cette expérience avait pour objectif de les initier à:

- diagnostiquer le niveau du tutorế et ses vrais problèmes d'apprentissage,

- à expliciter les représentations du tutoré, supposées responsables de certaines pratiques peu efficaces;

- la recherche-action - en menant une observation consciente de l'impact de ses propres interventions pédagogiques (et à en tenir un registre systématique sous forme d'un journal d'observations et de réflexions);

- à rédiger un rapport d'expérience.

Les rapports et l'évalution post-expérience ont montré un apport formatif très positif au niveau didactique. Les étudiants ont fait état d'une capacité d'observation et d'analyse accrue, ils ont témoigné de plus d'intérêt pour des études de type qualitatif, et surtout ils ont fait preuve d'une meilleure compréhension de la complexité présente dans toute situation d'enseignement / apprentissage. Ils ont également montré plus de facilité à identifier les problèmes et à les interprêter sans se retrancher derrière des explications superficielles (motivation insuffisante, étudiant peu doué, ou encore trop distrait...). Une dizaine de travaux de maîtrise très intéressants ( $\mathrm{y}$ compris pour leurs auteurs...) ont pris pour thème différents aspects de l'apprentissage, en mariant dans leur approche le théorique et le pratique dans le cadre d'une étude centrée sur l'exécution d'un type de tâche communicative personnelle.

\section{CONCLUSION: PEUT-ON NE PAS AUTONOMISER L'ÉTUDIANT AVANCÉ?}

Nous avons essayé ci-dessus de montrer brièvement les raisons - théoriques et pratiques - qui nous ont mené à élaborer et à mettre en oeuvre une démarche d'autonomisation pour les trois premières années d'études françaises (philologie romane), avec l'objectif majeur de préparer l'étudiant à continuer à se former lui-même, selon ses besoins et ses ambitions. La conséquence la plus visible a été de dépasser, tant pour l'étudiant que pour l'enseignant, la pédagogie se fondant sur le trio: mémorisation - répétition - automatisation, censée utile tout au plus pour préparer l'étudiant à réussir un test classique sous forme d'un ensemble d'exercices de langue, mais rendant problématique tout succès en communication réelle.

L'autonomisation vise précisément:

- à ne pas creuser un fossé entre les situations d'exercice et celles de communication, chaque type de situations correspondant à une charge et une élaboration - cognitive, émotionnelle et organisationnelle - différentes quantitativement et qualitativement, selon leur inscription dans le projet personnel; 
- à amener l'étudiant à pratiquer une corrélation, voire.une harmonie, entre ce qu'il pense de la communication exolingue et de sa maîtrise, ce que sont ses manières les plus efficaces d'apprendre et ce que peut être sa pratique «éclairée» de la LE.

La démarche d'autonomisation nous semble tout aussi intêressante sur le plan de la professionnalisation de futurs enseignans, notamment pour ce qui est de les sensibiliser à la complexité de la relation pédagogique dans une perspective interdisciplinaire, en les orientant vers une pratique enseignante réflexive et innovante à la fois, tout ceci au service du développement de la compétence personnelle du principal intéressé, c.-à-d. de l'apprenant.

\section{BIBLIOGRAPHIE}

Obrębska M. (1997), Les attentes des étudiants envers le tutorat en langue étrangère. Mémoire de maîtrise non publié. Université de Poznań - Institut de philologie romane.

Wilczyńska W. (1998), Une expérience d'apprentissage en semi-autonomie au niveau avancé: principes et bilan provisoire. Glottodidactica XXVI, pp. 277-285.

Wilczyńs ka W. (1999), Uczyć się czy być nauczanym. O autonomii w przyswajaniu języka obcego, Warszawa: PWN.

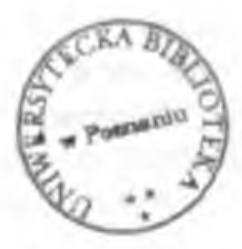

